\title{
56.
}

\section{DEMONSTRATION OF A GEOMETRICAL THEOREM OF JACOBI'S}

[From the Cambridge and Dublin Mathematical Journal, vol. III. (1848), pp. 48-49.]

THE theorem in question (Crelle, t. XII. [1834] p. 137) may be thus stated:

"If a cone be circumscribed about a surface of the second order, the focal lines of the cone are generating lines of a surface of the second order confocal to the given surface and which passes through the vertex of the cone."

Let $(\alpha, \beta, \gamma)$ be the coordinates of the given point,

$$
\frac{x^{2}}{a^{2}}+\frac{y^{2}}{b^{2}}+\frac{z^{2}}{c^{2}}=1
$$

the equation to the given surface. The equation of the circumscribed cone referred to its vertex is

$$
\left(\frac{x^{2}}{a^{2}}+\frac{y^{2}}{b^{2}}+\frac{z^{2}}{c^{2}}\right)\left(\frac{\alpha^{2}}{a^{2}}+\frac{\beta^{2}}{b^{2}}+\frac{\gamma^{2}}{c^{2}}-1\right)-\left(\frac{\alpha x}{a^{2}}+\frac{\beta y}{b^{2}}+\frac{\gamma z}{c^{2}}\right)^{2}=0,
$$

whence it is easily seen that the equation of the supplementary cone (i.e. the cone generated by lines through the vertex at right angles to the tangent planes of the cone in question) is

$$
(\alpha x+\beta y+\gamma z)^{2}-a^{2} x^{2}-b^{2} y^{2}-c^{2} z^{2}=0 .\left(^{1}\right)
$$

Suppose we have identically

$$
(\alpha x+\beta y+\gamma z)^{2}-a^{2} x^{2}-b^{2} y^{2}-c^{2} z^{2}-h\left(x^{2}+y^{2}+z^{2}\right)=(l x+m y+n z)\left(l^{\prime} x+m^{\prime} y+n^{\prime} z\right) ;
$$

$l x+m y+n z=0$. will determine the direction of one of the cyclic planes of the supplementary cone, and hence taking the centre for the origin the equations of the focal lines of the circumscribed cone are

$$
\frac{x-\alpha}{l}=\frac{y-\beta}{m}=\frac{z-\gamma}{n} .
$$

$1 A x^{2}+B y^{2}+C z^{2}+2 F y z+2 G x z+2 H x y=0$ being the equation of the first cone, that of the supplementary cone is $\mathbb{A} x^{2}+\mathbb{b} y^{2}+\mathfrak{C} z^{2}+2 \sqrt{5} y z+2 \mathfrak{G} z x+2$ 㨁 $x y=0$, these letters [denoting the inverse coefficients $B C-F^{2}$, \&c.]. 
It only remains therefore to determine the values of $l, m, n$ from the last equation but one. The condition which expresses that the first side of this equation divides itself into factors is easily reduced to

$$
\frac{a^{2}}{a^{2}+h}+\frac{\beta^{2}}{b^{2}+h}+\frac{\gamma^{2}}{c^{2}+h}=1
$$

Next, since the equation is identical, write

$$
x=\frac{\alpha}{a^{2}+h}, \quad y=\frac{\beta}{b^{2}+h}, \quad z=\frac{\gamma}{c^{2}+h},
$$

we deduce

$$
0=\frac{l \alpha}{a^{2}+h}+\frac{m \beta}{b^{2}+h}+\frac{n \gamma}{c^{2}+h}
$$

Again, putting

$$
x=\frac{l}{a^{2}+h}, \quad y=\frac{m}{b^{2}+h}, \quad z=\frac{n}{c^{2}+h},
$$

the whole equation divides by

$$
\left(\frac{l l^{\prime}}{a^{2}+h}+\frac{m m^{\prime}}{b^{2}+h}+\frac{n n^{\prime}}{c^{2}+h}+1\right)
$$

a factor whose value is easily seen to be $=-1$. And rejecting this, we have

$$
\frac{l^{2}}{a^{2}+h}+\frac{m^{2}}{b^{2}+h}+\frac{n^{2}}{c^{2}+h}=0
$$

Thus of the three equations (A), (B), and (C), the first determines $h$, and the remaining two give the ratios $l: m: n$. It is obvious that

$$
\frac{x^{2}}{a^{2}+h}+\frac{y^{2}}{b^{2}+h}+\frac{z^{2}}{c^{2}+h}=1
$$

is the equation of the surface confocal with the given surface which passes through the point $(\alpha, \beta, \gamma)$. The generating lines at this point are found by combining this equation with that of the tangent plane at the same point, viz.

$$
\frac{a x}{a^{2}+h}+\frac{\beta y}{b^{2}+h}+\frac{\gamma z}{c^{2}+h}=1
$$

and since these two equations are satisfied by $x=\alpha+l r, y=\beta+m r, z=\gamma+n r$, if $l, m, n$ are determined by the equations above, it follows that the focal lines of the cone are the generating lines of the surface, the theorem which was to be demonstrated. It is needless to remark that of the three confocal surfaces, the hyperboloid of one sheet has alone real generating lines; this is as it should be, since a cone has six focal lines, of which four are always imaginary. 